\title{
Depression Accelerates Tumor Cell Proliferation Via Regulating Serotonin/miR-144 Axis in NSCLC Mice
}

\section{Guangquan $\mathrm{Xu}^{1}$, Yongchao $\mathrm{Li}^{1}$, Ruidong $\mathrm{Zhu}^{1}$, Pengcheng Song ${ }^{1}$, Youlei Wei $^{1}$, Tian Liang ${ }^{2}$ and Tianze Zhang ${ }^{1 *}$}

${ }^{1}$ Department of Thoracic Surgery, The $2^{\text {nd }}$ Affiliated Hospital of Harbin Medical University, China

${ }^{2}$ Department of Obstetrics and Gynaecology, The $1^{\text {st }}$ Affiliated Hospital of Harbin

Medical University, China

*Corresponding Author: Tianze Zhang, Department of Thoracic Surgery, the $2^{\text {nd }}$

Affiliated Hospital of Harbin Medical University, China.
Received: September 24, 2020

Published: November 28, 2020

(C) All rights are reserved by Tianze Zhang., et al.

\begin{abstract}
Non-small cell lung cancer is known as a malignant tumor with low survival rate and poor prognosis. Depression affects various diseases. However, the effect of depression on the progression of NSCLC remains unclear. In our current study, chronic mild stress (CMS) mice was used as depression animal model. Depression prompted the tumor progression in vivo analysis, including increasing tumor indexes and reducing survival rate. Serotonin secretion was observed to be remarkable elevation in both serum and tumor tissue, which was positively related with tumor progression. In vitro assays, serotonin promoted the proliferation of A549 cells. Additionally, we observed that miR-144 expression was significantly downregulated in serotonin stimulated group. Further loss-of- and gain-of-function assays verified that miR-144 was the downstream factor of serotonin underlying the condition of CMS. Taken together, our research indicated that CMS-induced serotonin secretion accelerates NSCLC proliferation via inhibiting miR-144 expression, suggesting the potential therapeutic direction in NSCLC patients.
\end{abstract}

Keywords: Depression; Chronic Mild Stress; miR-144; NSCLC

\section{Introduction}

Non-small cell lung cancer (NSCLC) threatens patients with high morbidity and mortality with low 5-year survival [1]. It has been reported that around $85 \%$ patients with lung cancer were diagnosed as NSCLC [2], suggesting its urgent need in promising therapeutic treatment. Various risk factors accelerates the progression of NSCLC, promoting its tumor proliferation, including inflammation [3], oxidation [4], and also, the psychiatric factors, such as depression $[5,6]$.

Depression is generally induced by continuous chronic stress. Long-term exposure to chronic stress causally leads to various physio- and pathological changes from the central nervous system to peripheral organs $[7,8]$. Ample researches have documented positive relationship between depression and the risk of ischemic or coronary heart disease, causally increasing their morbidity and mortality [9]. Depression is also considered as a chronic, inflammation-related disorder, abnormally altering immune system, oxidative reaction and nitrosative stress [10]. In addition, the link between depression and chronic obstructive pulmonary disease has been well addressed [11], emphasizing the critical role of depression in chronic disease. Currently, depression has been gradually considered as the pro-carcinoma factor, which is the most malignant chronic diseases nowadays. Increasing studies have provided an overall view of depression-related carcinoma progression [1215]. However, there still little research on NSCLC with depression 
exist. More efforts are in requirements with this direction. The mechanisms underlying depression-induced disease progression step in the developing level.

Serotonin has been recognized as an important emotion-related factor, participating in various regulation of diseases. Researches have described serotonin as a biomarker and potential target against heart failure [16,17]. Additionally, its regulatory role beyond neurotransmitter has also been discovered. Serotonin attenuates LPS-induced systemic inflammation [18], as well as intestinal inflammation [19]. For carcinoma, serotonin caused a dose-dependent increase in the proliferation of bladder [20] and prostate cancer cells [21]. However, the effect of serotonin on NSCLC remains unclear. Based on these theories, our study was designed to investigate the relationship between depression, serotonin and NSCLC, providing a novel therapeutic direction and strategy against NSCLC.

\section{Materials and Methods}

Animals

Animal experimental protocols were consented by the Ethic Committees of Harbin Medical University, and were in accordance with the Guide for the Use and Care of Laboratory Animals published by US National Institutes of Health (NIH Publication No. 85-23, revised 1996). NSCLC tumor-bared mice were established as previous study [22]. In brief, six-week old BALB/c nude mice, which were purchased from the Animal Center of the $2^{\text {nd }}$ Affiliated Hospital of Harbin Medical University (Harbin, China). All mice were housed in a dedicated room (12h dark/light cycle, controlled temperature at $22 \pm 1^{\circ} \mathrm{C}$, constant humidity at $55 \pm 5 \%$ ) for 1 -week acclimatization. According to the experimental design, nude mice bearing A549 tumor xenografts were divided into following groups randomly: controls, CMS, CMS+apocynin, CMS+SSRI, serotonin, serotonin+miR-144, and serotonin+negative control (NC). The volumes of the tumors were measured every week for 3 month.

For CMS animals were obtained as a gift from substance-dependent laboratory of Qiqihar Medical University. As shown in previous study [23], CMS includes limited room restraint, forced warm water bath, water/food deprivation, housing in wet saw dust, and reversed day/night cycle, etc.

Serotonin was treated as previously documented [24]. In brief, serotonin (Sigma-Aldrich Co., Ltd, Louis, MO, USA) was administrated via oral gavage (7.5 mg/kg/day). Apocynin (5 mg/kg/day, Calbiochem, Gibbstown, NJ) was administrated orally, dissolved in drink water [25]. SSRI (10 mL/kg fluoxetine dissolved) was injected intraperitoneally, dissolved in $0.9 \%$ saline $(0.9 \% \mathrm{NaCl}, \mathrm{pH} 7.4)$ accordingly [26]. Controls received the same volume of dissolved solution.

\section{Cell culture and treatment}

NSCLC cell line, A549 cells, was purchased from Shanghai Institutes for Biological Sciences (SIBS, China). Standard culture medium includes the RPMI-1640 medium (Thermo Fisher Scientific, Waltham, MA, USA), 10\% fetal bovine serum (FBS, Gibco, USA) and $1 \%$ penicillin/streptomycin $(100 \mu \mathrm{g} / \mathrm{mL})$. Cells were incubated in $5 \% \mathrm{CO}_{2}$ at $37^{\circ} \mathrm{C}$. Before experiments, cells were staffed overnight in FBS-free restriction/treatment medium.

In vitro assays, serotonin ( $5 \mu \mathrm{M})$ and SSRI (10 $\mu \mathrm{M}$ fluoxetine hydrochloride, Sigma-Aldrich) were dissolved in dimethyl sulfoxide (DMSO) according to previous study [27,28].

\section{Cell transfection}

A549 cells were seeded into 6-well plate at a density of $2 \times 10^{6}$ cells/well. At the confluence of $80 \%$, cells were starved in serumfree medium overnight before transfection. miR-144, mutated miR-144 (negative control, NC), anti-miR-144 antisense oligoribonucleotide (AMO-144), and AMO-NC (RiboBio Co., Ltd, Guangzhou, China) were transfected into A549 cells as experimental design. For the protocol of transfection, A549 cells were cultured with serum-free medium for $12 \mathrm{~h}$. miRNAs and lipofectamine 2000 (Invitrogen, Carlsbad, USA) were mixed for 5 minutes prior to transfection. Then, the two mixtures were combined and incubated at room temperature for 15 minutes and finally, the mixture was added to A549 cells. The transfection medium was replaced by regular growth medium after $6 \mathrm{~h}$ transfection. as previous study showed.

\section{Enzyme-linked immunosorbent assay}

Serum contents of serotonin (serotonin; BOSTER, Wuhan, China) were determined using an ELISA kit following the manufacturer's instructions.

\section{Western blot}

Protein samples (NSCLC tumor tissues) were extracted and dissolved with RIPA buffer (Solarbio, Beijing, China) with protease inhibitors (Sigma, Louis, MO, USA). BCA (Beyotime, Shanghai, China) method was used to quantify the concentration. SDS-PAGE (10\% polyacrylamide gels) was used to separate proteins with different molecular weight, and then transferred to nitrocellulose 
membrane. Non-fat milk (5\%) was used for blocking subsequently, and the membranes were incubated with the primary antibodies for serotonin (ab66047, Abcam, USA) and GAPDH (TA-08, Zhongshan Golden Bridge Biotechnology, Beijing, China), which was used as internal control. After overnight incubation, florescence-labeled secondary antibody was incubated in dark. Odyssey Infrared Imaging System (LI-COR, Lincoln, NB, USA) was used to calculate the relative expression level of serotonin compared with the internal control. which GAPDH was used as internal control.

\section{Real-time PCR}

Total RNA was harvested from A549 cells via TRIzol reagent (Invitrogen, Carlsbad, CA, USA). cDNA synthesis was performed using a High-Capacity cDNA Reverse Transcription Kit (Applied Biosystems, Carlsbad, CA, USA; Cat. no.4368814). The levels of serotonin and miR-144were determined by SYBR Green I incorporation method and ABI 7500 fast real-time PCR system (Applied Biosystems, USA). GAPDH and U6 were used as internal control, respectively.

\section{Proliferation assay}

Proliferation detection of A549 cells was conducted by CCK- 8 assay. $100 \mu \mathrm{L}$ medium containing A549 cells were seeded in a 96-well plate before detection. CCK-8 reagent was added to each well (10 $\mu \mathrm{L} /$ well). Cells were incubated for $4 \mathrm{~h}$ in cell-culture circumstance. Then, the optical density (OD) value (450 nm) was determined by an enzyme-linked immunosorbent assay plate reader (Bioreader).

\section{Statistical analysis}

All values were presented as mean \pm S: E: M: Statistical comparisons were performed by Student's t-test between two groups or one-way ANOVA for multiple comparisons. $\mathrm{p}<$ 0:05 was considered to indicate a significant difference. Data were analyzed using the GraphPad Prism 7.0 software. Correlations between miR-144 and serotonin were assessed by using Pearson, Spearman, and Kendall's rank correlation coefficient analyses [22].

\section{Results}

Depression positively related with malignant ending in NSCLC mice

Depression plays vital roles on the progression of various diseases, promoting the deterioration, and finally leads to a bad end. To detect the influence of depression on NSCLC, we established NSCLC with CMS, and further investigate the criteria of tumor progression, including tumor size, volume, weight, and survival rate. Apocynin is commonly recognized as an anti-depression drug, which we used as the positive control group. The result of Figure 1 shows that CMS remarkably increased the values of tumor size (Figure 1A), volume (Figure 1B), and weight (Figure 1C), reducing the survival rate (Figure 1D) compared non-CMS stimulated controls and Apocynin treated CMS group. These data indicated that depression is positively related with the progression of NSCLC, emphasizing the importance tote further detect the underlying mechanism.

B

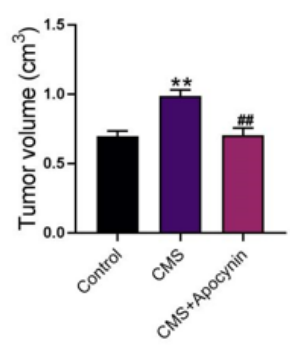

D

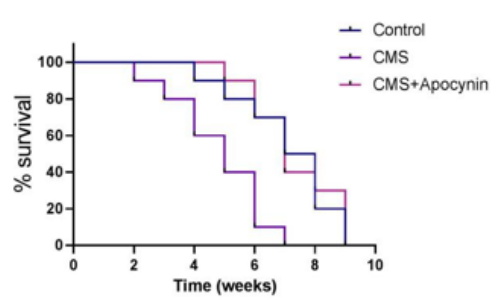

Figure 1: Depression induced by CMS promotes the progression of NSCLC. (A-D) Tumor size, volume, weight and survival rate were continuously recorded and calculated 3 months after animal model establishment. $\mathrm{N}=10$ in each batches. ${ }^{* *} \mathrm{p}<0.01,{ }^{* * *} \mathrm{p}<0.001$. Compared with controls, \#\#p < 0.01, \#\#\#p < 0.001 compared with CMS group. 
Serotonin involved in depression-related NSCLC tumor progression

Serotonin is a key regulatory factor on emotion, especially on depression. To gain the insight of the mechanism, we examined the levels of serotonin in serum and tumor tissue homogenate. The results show that serotonin was significantly elevated in CMS group, whereas repressed after Apocynin treatment (Figure 2A-2C). To detect the relationship between serotonin and NSCLC tumor progression, we treated CMS mice with selective serotonin reuptake inhibitor (SSRI). The results show that serotonin inhibition by SSRI ameliorated the condition of NSCLC, including the tumor size (Figure 2D), tumor volume (Figure 2E), tumor weight (Figure 2F), as well as survival rate (Figure $2 \mathrm{G}$ ). Relationship analysis results show that serotonin is positively related with the progression of NSCLC (Figure 2H-2J), suggesting its regulatory role of NSCLC.
A

D
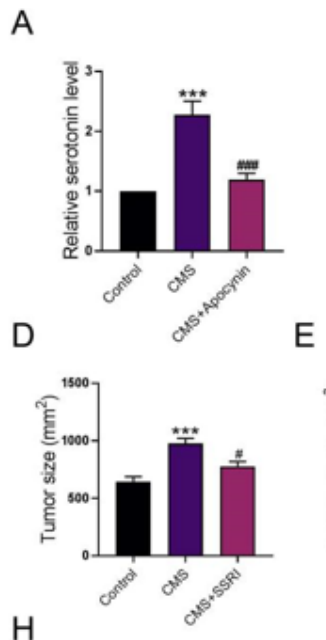

$\mathrm{H}$

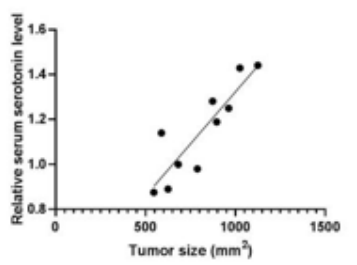

B
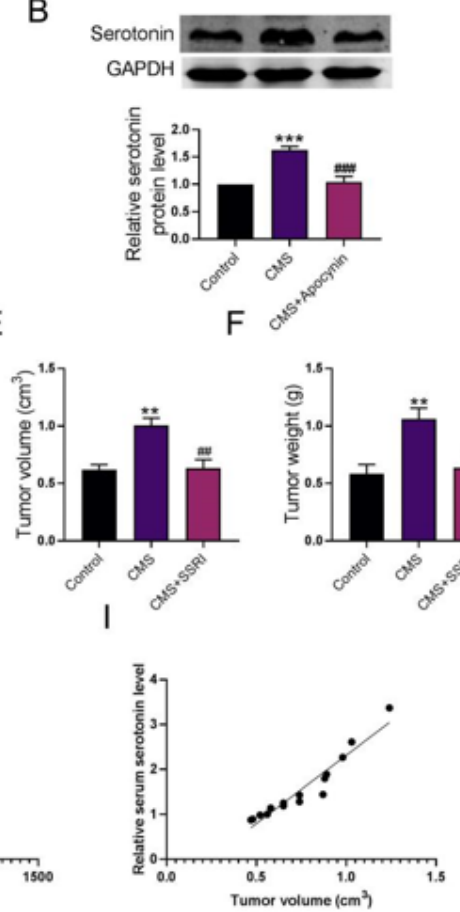

C
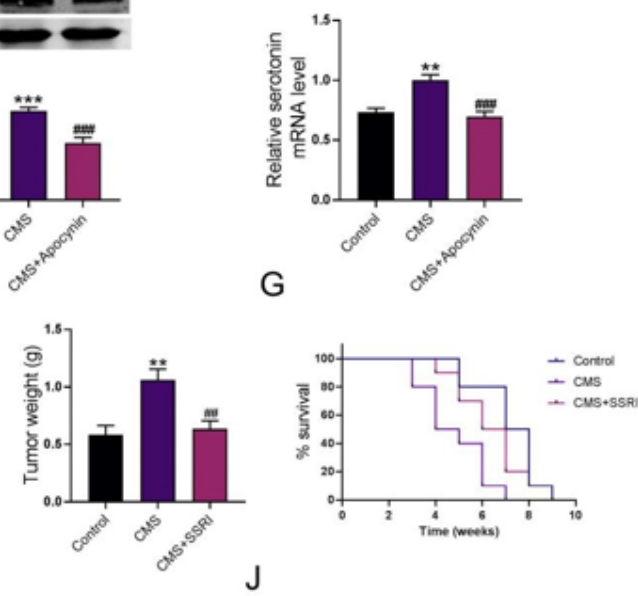

$J$

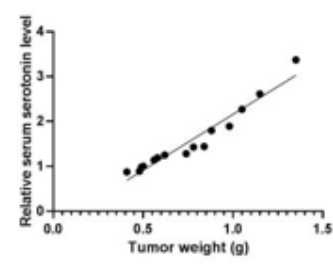

Figure 2: Serotonin is positively related with depression-affricated tumor progression of NSCLC. (A) Serotonin level was examined using ELISA kit in mice serum. $\mathrm{n}=5$ in each group, ${ }^{* * *} \mathrm{p}<0.001$ compared with control group, \#\#\# $<0.001$ compared with CMS group. (B-C) Serotonin levels were detected by real-time PCR and western blot in mice tissue homogenate, respectively. $\mathrm{n}=5$, ${ }^{* *} \mathrm{p}<0.01,{ }^{* * *} \mathrm{p}<$ 0.001 compared with control group, \#\#\#p < 0.001 compared with CMS group. (D-G) Tumor size, volume, weight and survival rate were recorded and calculated in control, CMS and CMS+SSRI groups. $\mathrm{n}=5$ in each batches, ${ }^{* *} \mathrm{p}<0.01,{ }^{* * *} \mathrm{p}<0.001$ compared with control group, \#p < 0.05, \#\#p < 0.01 compared with CMS group. (H-J) Relationship between serum serotonin levels and tumor index (tumor size, volume and weight), $\mathrm{X}$ axis represents relative tumor index, and $\mathrm{Y}$ axis represents relative serum serotonin levels, $\mathrm{n}=10 \mathrm{in}$ each batches.

Serotonin promotes the progression of NSCLC via downregulating miR-144

Previous studies have showed that miR-144 is not only participates in the proliferation of NSCLC cells [29,30], but also regulates depression-related physiological processes [31-33]. Based on these researches, we further verified the underlying mechanism with the involvement of miR-144. A549 cells were used as in vitro materials. The proliferation of A549 cells was measured as figure 3A and 
3B show. Compared with controls, serotonin stimulates A549 cell proliferation, increasing the cell number (Figure 3A and 3B). Additionally, miR-144 was reduced in serotonin stimulated group, as well as CMS group (Figure 3C and 3D), suggesting the regulatory role of serotonin on miR-144. To further investigating the effect of
serotonin/miR-144 on cell proliferation of A549, we used gain- and loss-of-function approaches. The results reveal that miR-144 is the downstream factor of serotonin, participating the regulation of serotonin on cell proliferation of A549 (Figure 3E and 3F).
A

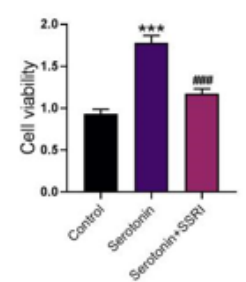

$\mathrm{E}$

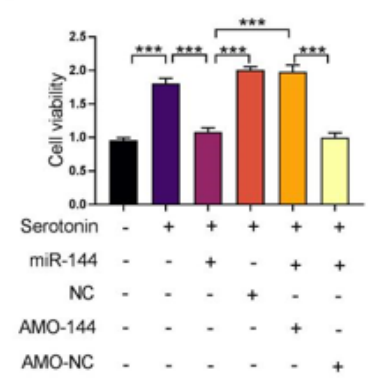

B

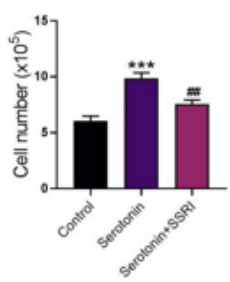

C

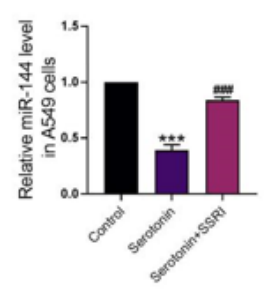

$\mathrm{F}$

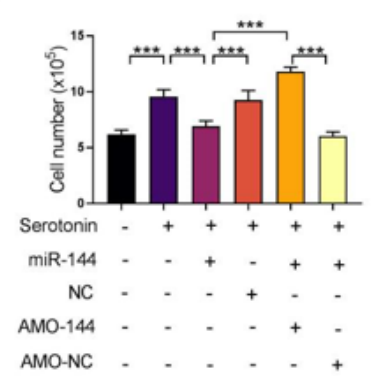

Figure 3: miR-144 inhibits A549 cell proliferation as the downstream factor of serotonin. (A, B) A549 cell viability and number were detected by CCK-8 and trypan blue assay, respectively. $\mathrm{N}=5$ in each group, ${ }^{* *} \mathrm{p}<0.01,{ }^{* * *} \mathrm{p}<0.001$ compared with controls, \#\#p $<0.01$, $\# \# \#$ p 0.001 compared with serotonin group. (C, D) The levels of miR-144 in A549 cells and tumor tissue homogenate were detected

by real-time PCR, calibrated by U6 expression. $n=5,{ }^{* * *} p<0.001$ compared with controls, \#\#\#p $<0.001$ compared with serotonin group. (E-F) Cell viability and number were measured by CCK- 8 and trypan blue assays in different groups, $\mathrm{n}=5$ in each batches, ${ }^{* * *} \mathrm{p}<$ 0.001 .

Serotonin/miR-144 axis regulates depression-related tumor progression in NSCLC mice

According to the in vitro results, we then verified the effect of serotonin/miR-144 on depression-induced NSCLC aggravation by in vivo analysis. A549 cells were transfected with miR-144 mimics, as well as its mutated vectors (NC) before transferred into mice. After serotonin administration, tumor indexes were measured as figure
4A-4D show. Overexpression of miR-144 in A549 mice significantly reduced the tumor size, volume and weight, ameliorating the survival rate (Figure 4A-4D). Similarly, miR-144 shows the beneficial effects on tumor progression in CMS stimulated mice (Figure 4E$4 \mathrm{H}$ ). These data indicate that serotonin/miR-144 regulates depression-promoted tumor progression of NSCLC, suggesting a potential therapeutic target against NSCLC. 
A

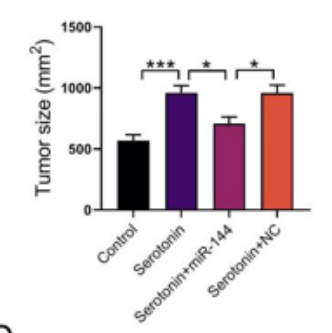

D

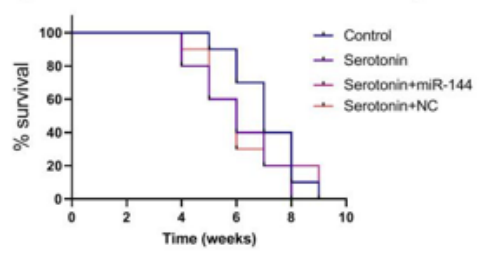

F

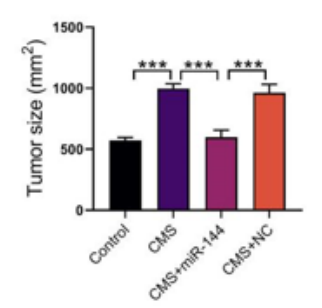

B

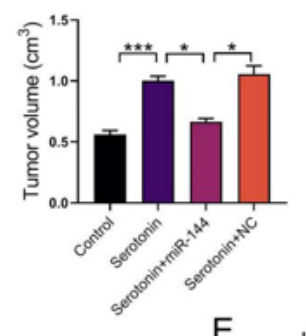

C

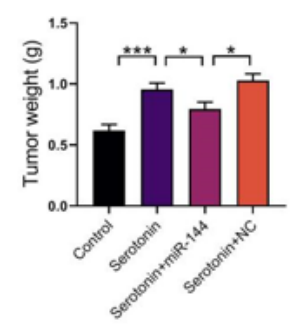

E

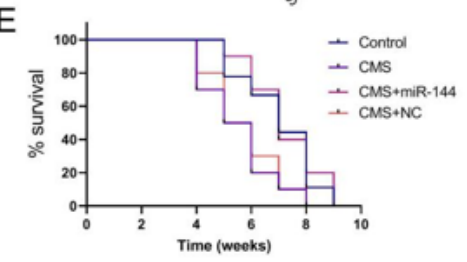

G

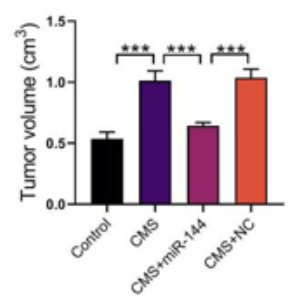

$\mathrm{H}$

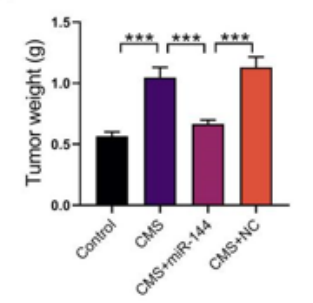

Figure 4: Serotonin/miR-144 ameliorates tumor progression of NSCLC induced by depression. Tumor indexes, including tumor size, volume, weight and survival rate were recorded and calculated in different groups. $\mathrm{n}=10$ in each group, ${ }^{*} \mathrm{p}<0.05,{ }^{* * *} \mathrm{p}<0.001$.

\section{Discussion}

Our research has observed that 1) Depression promotes the progression of NSCLC, and 2) serotonin, at least in part, participates in depression-induced NSCLC aggravation. 3) miR-144 is the downstream effector of serotonin and 4) serotonin/miR-144 axis is the key regulatory pathway, mediating depression accelerated NSCLC cell proliferation. We have analyzed the multiple pathogenic factor of NSCLC, and uncovered the influence of psychiatric factor, as well as the underlying mechanism in this carcinoma. In this case, this study provides not only the discovery in therapeutic strategy and potential targets of NSCLC, but also initiates the cross-disciplinary direction for NSCLC treatment.

Nowadays, lung cancer remains the leading cause of mortality worldwide, especially NSCLC, possessing the most common proportion of cases. Although multiple treatments in development, including such as thoracic surgery, chemotherapy, radiotherapy and targeted drug treatments, the survival rate of 5 year, as well as the prognostic result is still under-satisfied. Tumor cell proliferation contributes to the main malignancy in NSCLC, requiring the effective approach to block. Signalling pathways involved in the proliferation of NSCLC tumor cells includes Wnt pathway [34], NFКB pathway [35], and E2F2 pathway [36], et al. Researches have also highlighted the importance of miRNAs in this process, and discovered the regulatory effect of Notch-1/miR-137, as well as PDCD4/miR-421 axis on NSCLC proliferation [37,38]. Additionally, miR-124 has been reported to exert its beneficial effect on NSCLC $[39,40]$, recognized as a tumor suppressor and prognostic marker. Based on these theories, researches of NSCLC focused on miR-124 are of great value of clinical treatments.

While plenty of the pathogenesis of NSCLC, the psychiatry factor of depression remains an obscure step. Physical uncomfortableness from illness and treatments, as well as organ functional 
impairment adversely promote the formation of depression. In turn, depression adversely accelerates the progression of chronic illnesses. The mortality rate in cardiac infarction patients with depression is remarkably elevated compared with normal mental condition patients (26\% vs 7\%) [41]. Researches have also revealed depression as an independent risk factor of heart failure, threatening the life-span of patients with cardiovascular diseases [42]. Long-term pressure and anxiety from illness, family, and medical cost in NSCLC patients frequently contribute to depression. NSCLC has been identified to be the highest rates of co-morbid depression among all cancer types. Studies focused on NSCLC and depression has showed that NSCLC patients with mutant EGFR is negatively associated with depression [5]. Also, depression is related with worse survival in patients with newly diagnosed NSCLC [43]. Although several researches exist, the underlying reason for this association, together with its mechanism is not entirely clear, raising the requirement for further detection.

Serotonin is a biogenic monoamine, characterized as a neuromodulatory factor regulating neoplastic capabilities. Additionally, it also acts as the local mediator in the gut and vasoactive agent in the blood, exerting its biological effects via interacting with receptors, as well as multiple pathways [44]. The biological underpinning of serotonin on depression is becoming increasingly understood. Serotonin participates in the development of neuronal networks, and its dysfunction thereby contributes to brain disorders. The relationship between serotonin and the pathophysiology of depression has been well reviewed [45], however, the biological relevance, triggers and molecular mechanisms are only beginning to be understood. Previous study has revealed the growth inhibition of prostatic carcinoma by serotonin antagonists [46], and serotonin activates MAP kinase and PI3K/Akt signaling pathways in the progression of prostate cancer [47]. In addition, serotonin has been observed to be involved in of small cell lung carcinoma cells, colonic adenocarcinoma, breast carcinoma, and Bladder carcinoma., et al. [44], whereas the relationship between serotonin and NSCLC remains incomprehensive. Thus, our study has initially uncovered this relationship, providing the novel therapeutic direction against NSCLC.

Importance of miRNAs has been recognized in various physiopathological processes. Researches of miRNAs focused on carcinoma have been gradually emphasized. The development of gastric cancer has been reported to be regulated by miR-183 via LncRNA MALAT1/miR-183/SIRT1 axis and PI3K/AKT/mTOR signals [48]. MiR-135a-5p promotes lung cancer progression via targeting LOXL4 [49]. MiR-155/miR-143 axis participates in TGF- $\beta 1$ promoted colorectal cancer immune escape [50]. The involvement of miR-150/ $\beta$-catenin axis in colorectal cancer progression has also been identified recently [51]. Among these miRNAs, miR-144 has been considered as a key regulator on cervical cancer [52], gastric cancer [53], colorectal cancer [54], as well as NSCLC. Studies have addressed the inhibitory effects of miR-144 on radiosensitivity of NSCLC via regulating ATF2 [30] and cancer proliferation by targeting CDKL1 [55]. According to previous studies, miR-144 is also tightly related with depression. Expression level of miR-144-5p is significantly downregulated in depressive patients [31], suggesting its potential peripheral biomarker for pathologic processes related to depression. Our current study has verified that miR-144 mediates depression-promoted NSCLC progression via inhibiting cell proliferation, indicating the clinical potential of miR-144 in psychiatric-combined carcinoma treatment.

\section{Conclusion}

In this study, we found that depression is positively related with malignancy of NSCLC via elevating serotonin expression. Serotonin/miR-144 axis regulates depression-promoted NSCLC progression via inhibiting cancer cell proliferation property. Future investigations are needed to verify the downstream targets of miR144 and define the specific serotonin receptor involved in the process. Also, the resource of serotonin and the secretion mechanisms are still in requirement, raising the need of joint contribution cross disciplines.

\section{Acknowledgements}

This work was supported by the Funds for Scientific Research Project of the Health and Family Planning Commission of Heilongjiang Province (2018506) and the Heilongjiang Postdoctoral Fund (LBH-Z18215).

\section{Conflict of Interests}

The authors declare no conflict of interests.

\section{Data Available}

The datasets used and/or analyzed during the current study are available from the corresponding author on reasonable request. 


\section{Bibliography}

1. Herbst RS., et al. "The biology and management of non-small cell lung cancer". Nature 553.7689 (2018): 446-454.

2. Relli V., et al. "Abandoning the Notion of Non-Small Cell Lung Cancer". Trends in Molecular Medicine 25.7 (2019): 585-594.

3. Zhao C., et al. "IL-17 induces NSCLC A549 cell proliferation via the upregulation of HMGA1, resulting in an increased cyclin D1 expression". International Journal of Oncology 52.5 (2018): 1579-1592.

4. Meng L., et al. "Mitochondrial NDUFA4L2 protein promotes the vitality of lung cancer cells by repressing oxidative stress". Thoracic Cancer 10.4 (2019): 676-685.

5. McFarland DC., et al. "Depression and inflammation among epidermal growth factor receptor (EGFR) mutant nonsmall cell lung cancer patients". Psychoncology 28.9 (2019): 14611469.

6. Jacobs JM., et al. "Depression, inflammation, and epidermal growth factor receptor (EGFR) status in metastatic non-small cell lung cancer: A pilot study". Journal of Psychosomatic Research 99 (2017): 28-33.

7. Antoniuk S., et al. "Chronic unpredictable mild stress for modeling depression in rodents: Meta-analysis of model reliability". Neuroscience and Biobehavioral Reviews 99 (2019): 101116.

8. Ross RA., et al. "The Role of Chronic Stress in Anxious Depression". Chronic Stress (Thousand Oaks) 1 (2017): 2470547016689472.

9. Headrick JP., et al. "The heartbreak of depression: 'Psycho-cardiac' coupling in myocardial infarction". Journal of Molecular and Cellular Cardiology 106 (2017): 14-28.

10. Berk M., et al. "So depression is an inflammatory disease, but where does the inflammation come from?" BMC Medicine 11 (2013): 200.

11. Nagaich AK and Misra K. "Highly efficient synthesis of oligodeoxyribonucleotides using alpha-phenyl cinnamoyl group for selective amino protection". Nucleic Acids Research 17.13 (1989): 5125-5134.

12. Sotelo JL., et al. "The biology of depression in cancer and the relationship between depression and cancer progression". International Review of Psychiatry 26.1 (2014): 16-30.
13. Cvetković J and Nenadović M. "Depression in breast cancer patients". Psychiatry Research 240 (2016): 343-347.

14. Yeung KS., et al. "Herbal medicine for depression and anxiety: A systematic review with assessment of potential psycho-oncologic relevance". Psychiatry Research 32.5 (2018): 865-891.

15. Barreto FS., et al. "Tryptophan catabolites along the indoleamine 2,3-dioxygenase pathway as a biological link between depression and cancer". Behavioural Pharmacology 29 (2018): 165-180.

16. Selim AM., et al. "Plasma Serotonin in Heart Failure: Possible Marker and Potential Treatment Target". Heart, Lung and Circulation 26.5 (2017): 442-449.

17. Aloisi G., et al. "Depression and heart failure: an intricate relationship". Monaldi Archives for Chest Disease 89.3 (2019).

18. Mota CMD., et al. "Central serotonin attenuates LPS-induced systemic inflammation". Brain, Behavior, and Immunity 66 (2017): 372-381.

19. Shajib MS., et al. "Diverse Effects of Gut-Derived Serotonin in Intestinal Inflammation". ACS Chemical Neuroscience 8.5 (2017): 920-931.

20. Siddiqui EJ., et al. "The effect of serotonin and serotonin antagonists on bladder cancer cell proliferation". BJU International 97.3 (2006): 634-639.

21. Siddiqui EJ., et al. "The role of serotonin (5-hydroxytryptamine1A and 1B) receptors in prostate cancer cell proliferation". Journal of Urology 176 (2006): 1648-1653.

22. Zhang T., et al. "Transcription Factor p53 Suppresses Tumor Growth by Prompting Pyroptosis in Non-Small-Cell Lung Cancer". Oxidative Medicine and Cellular Longevity (2019): 8746895.

23. Lv H., et al. "Chronic mild stress induced anxiety-like behaviors can Be attenuated by inhibition of NOX2-derived oxidative stress". Journal of Psychiatric Research 114 (2019): 55-66.

24. Park CH., et al. "Protective Effects of Serotonin and its Derivatives, N-Feruloylserotonin and N- (p-Coumaroyl) Serotonin, Against Cisplatin-Induced Renal Damage in Mice". American Journal of Chinese Medicine 47.2 (2019): 369-383.

25. Mitazaki S., et al. "Apocynin reduced doxycycline-induced acute liver injury in ovariectomized mice". Toxicology Reports 3 (2016): 357-363. 
26. Song L., et al. "Hippocampal PPAR $\alpha$ is a novel therapeutic target for depression and mediates the antidepressant actions of fluoxetine in mice". British Journal of Pharmacology 175.14 (2018): 2968-2987.

27. Cerulo G., et al. "Serotonin activates cell survival and apoptotic death responses in cultured epithelial thyroid cells". Biochimie 105 (2014): 211-5.

28. Mundalil Vasu M., et al. "Fluoxetine Increases the Expression of miR-572 and miR-663a in Human Neuroblastoma Cell Lines”. PLoS One 11.10 (2016): e0164425.

29. Chen YJ., et al. "Down-regulation of microRNA-144-3p and its clinical value in non-small cell lung cancer: a comprehensive analysis based on microarray, miRNA-sequencing, and quantitative real-time PCR data". Respiratory Research 20.1 (2019): 48.

30. Song L., et al. "miR-144-5p Enhances the Radiosensitivity of Non-Small-Cell Lung Cancer Cells via Targeting ATF2". Biomed Research International (2018): 5109497.

31. Wang X., et al. "Circulating microRNA-144-5p is associated with depressive disorders". Clinical Epigenetics 7.1 (2015): 69.

32. Ma K., et al. "The molecular mechanism underlying GABAergic dysfunction in nucleus accumbens of depression-like behaviours in mice". Journal of Cellular and Molecular Medicine 23.10 (2019): 7021-7028.

33. Short AK., et al. "Elevated paternal glucocorticoid exposure alters the small noncoding RNA profile in sperm and modifies anxiety and depressive phenotypes in the offspring". Translational Psychiatry 6.6 (2016): e837.

34. Stewart DJ. "Wnt signaling pathway in non-small cell lung cancer". Journal of the National Cancer Institute 106.1 (2014): djt356.

35. Zou J., et al. "Polydatin suppresses proliferation and metastasis of non-small cell lung cancer cells by inhibiting NLRP3 inflammasome activation via NF- $\kappa \mathrm{B}$ pathway". Biomed Pharmacotherapy 108 (2018): 130-136.

36. Li X., et al. "Circular RNA circPVT1 Promotes Proliferation and Invasion Through Sponging miR-125b and Activating E2F2 Signaling in Non-Small Cell Lung Cancer". Cellular Physiology and Biochemistry 51.5 (2018): 2324-2340.

37. Wang X., et al. "Knockdown of LncRNA-XIST Suppresses Proliferation and TGF- $\beta 1$-Induced EMT in NSCLC Through the Notch-1 Pathway by Regulation of miR-137". Genetic Testing and Molecular Biomarkers 22.6 (2018): 333-342.
38. Yang YN., et al. "MicroRNA-421 promotes proliferation and invasion of non-small cell lung cancer cells through targeting PDCD4". Pathology - Research and Practice 215.10 (2019): 152555.

39. Li S., et al. "The IncRNA MALAT1 contributes to non-small cell lung cancer development via modulating miR-124/STAT3 axis". Journal of Cellular Physiology 233.9 (2018): 6679-6688.

40. Li X., et al. "The tumor suppressor miR-124 inhibits cell proliferation by targeting STAT3 and functions as a prognostic marker for postoperative NSCLC patients". International Journal of Oncology 46.2 (2015): 798-808.

41. Romanelli J., et al. "The significance of depression in older patients after myocardial infarction". Journal of the American Geriatrics Society 50.5 (2002): 817-22.

42. Williams SA., et al. "Depression and risk of heart failure among the elderly: a prospective community-based study". Psychosomatic Medicine 64.1 (2002): 6-12.

43. Pirl WF, et al. "Depression and survival in metastatic nonsmall-cell lung cancer: effects of early palliative care. J Clin Oncol 30.12 (2012): 1310-1315.

44. Sarrouilhe D., et al. "Serotonin and cancer: what is the link?" Current Molecular Medicine 15.1 (2015): 62-77.

45. Kraus C., et al. "Serotonin and neuroplasticity - Links between molecular, functional and structural pathophysiology in depression". Neuroscience and Biobehavioral Reviews 77 (2017): 317-326.

46. Abdul M., et al. "Growth inhibition of human prostatic carcinoma cell lines by serotonin antagonists". Anticancer Research 14 (1994): 1215-1220.

47. Dizeyi N., et al. "Serotonin activates MAP kinase and PI3K/Akt signaling pathways in prostate cancer cell lines". Urologic Oncology 29.4 (2011): 436-445.

48. Li H., et al. "MicroRNA-183 affects the development of gastric cancer by regulating autophagy via MALAT1-miR-183-SIRT1 axis and PI3K/AKT/mTOR signals". Artificial Cells, Nanomedicine, and Biotechnology 47.1 (2019): 3163-3171.

49. Zhang Y., et al. "Downregulation of lysyl oxidase-like 4 LOXL4 by miR-135a-5p promotes lung cancer progression in vitro and in vivo". Journal of Cellular Physiology 234.10 (2019): $18679-18687$. 
50. Zhou X., et al. "TGF- $\beta 1$ promotes colorectal cancer immune escape by elevating B7-H3 and B7-H4 via the miR-155/miR-143 axis". Oncotarget 7.41 (2016): 67196-67211.

51. He Z., et al. "The involvement of miR-150/ $\beta$-catenin axis in colorectal cancer progression". Biomedicine and Pharmacotherapy 121 (2020): 109495.

52. Wu J., et al. "MiR-144-3p: a novel tumor suppressor targeting MAPK6 in cervical cancer". Journal of Physiology and Biochemistry 75.2 (2019): 143-152.

53. Tian QG., et al. "The role of miR-144/GSPT1 axis in gastric cancer". European Review for Medical and Pharmacological Sciences 22.13 (2018): 4138-4145.

54. Choi HH., et al. "Stool-Based miR-92a and miR-144* as Noninvasive Biomarkers for Colorectal Cancer Screening”. Oncology 97.3 (2019): 173-179.

55. Lu M., et al. "circRACGAP1 promotes non-small cell lung cancer proliferation by regulating miR-144-5p/CDKL1 signaling pathway". Cancer Gene Therapy (2020).

\section{Assets from publication with us}

- Prompt Acknowledgement after receiving the article

- Thorough Double blinded peer review

- Rapid Publication

- Issue of Publication Certificate

- High visibility of your Published work

Website: $\underline{w w w . a c t a s c i e n t i f i c . c o m / ~}$

Submit Article: www.actascientific.com/submission.php

Email us: editor@actascientific.com

Contact us: +919182824667 